\title{
Tratamento de fratura complexa de mandíbula em paciente vítima de acidentemotociclístico - relato de caso
}

\author{
Treatment of complex jaw fracture in a patient victim of a motorcycle accident - case report
}

Tratamiento de la fractura compleja de la mandíbula en un paciente víctima de un accidente

demotocicleta - reporte de caso

Recebido: 12/10/2021 | Revisado: 19/10/2021 | Aceito: 17/12/2021 | Publicado: 24/12/2021

\author{
Lara Fernanda Carlos Lima \\ ORCID: https://orcid.org/0000-0002-0695-7490 \\ Universidade Federal do Piauí, Brasil \\ E-mail: lara_lf1@hotmail.com \\ Francisco Antonio de Jesus Costa Silva \\ ORCID: https://orcid.org/0000-0002-8682-2237 \\ Universidade Federal do Piauí, Brasil \\ E-mail: franciscoo_@outlook.com \\ Aline Raquel de Sousa Nogueira \\ ORCID: https://orcid.org/0000-0002-2038-8415 \\ Universidade Federal do Piauí, Brasil \\ E- mail: alinerakelsn@gmail.com \\ Monize Kaylane Braz Pereira \\ ORCID: https://orcid.org/0000-0002-4916-8993 \\ Universidade Federal do Piauí, Brasil \\ E-mail: monizebraz@hotmail.com+ \\ Maria Cândida de Almeida Lopes \\ ORCID: https://orcid.org/0000-0002-2817-8949 \\ Universidade Federal do Piauí, Brasil \\ E-mail: candidalopes@ufpi.edu.br \\ Márcia Socorro da Costa Borba \\ ORCID: https://orcid.org/0000-0001-9193-1078 \\ Centro Universitário UniFacid, Brasil \\ E-mail: borbamarcia@hotmail.com
}

\begin{abstract}
Resumo
A mandíbula é o único osso móvel da face e possui função na mastigação, deglutição, fonação e estética facial. É fraturada comfrequência, possuindo origem variada, tendo prevalência de idade até os 40 anos, em casos de queda da própria altura é mais frequente em crianças e idosos. Paciente do sexo masculino, 64 anos, vítima de acidente motociclístico, compareceu ao serviço de Cirurgia e Traumatologia Bucomaxilofacial do Hospital de Urgência de Teresina. Ao exame clínico apresentava edema, lacerações e escoriações na face no lado direito, apresentou degrau ósseo à palpação de mandíbula em região de ângulo direito e sínfise. Ao exame de imagem, constatou-se, na tomografia computadorizada, a presença de uma descontinuidade do arco mandibular com múltiplos traços de fratura, em região de ângulo direito, corpo direito e sínfise da mandíbula esquerda. Fechandoo diagnóstico em Fratura complexa de mandíbula, encaminhando assim, a intervenção cirúrgica para redução e fixação da fratura. Utilizou- se quatro placas do sistema 2.0. Realizou-se a infiltração local com xilocaína com vasoconstritor $2 \%$, incisão para o acesso de Risdon (submandibular), exposição da fratura, reanatomização dos fragmentos ósseos e a osteossíntese com duas placas 2.0 e 15 parafusos em área de compressão e tensão, o mesmo foi feito no acesso intraoral na região de sínfise. Por fím, sutura por planos. O paciente teve alta hospitalar com um dia e seguiu com acompanhamento ambulatorial. A partir do caso apresentado, em casos de fraturas de mandíbula o acesso extra oral de risdon e o intraoral se mostram eficazes e seguros.
\end{abstract}

Palavras-chave: Fraturas mandibulares; Ossos faciais; Técnicas de fixação da mandíbula.

\begin{abstract}
The mandible is the only mobile bone in the face and has a role in mastication, swallowing, speech and facial esthetics. It is frequently fractured, having a varied origin, with a prevalence of up to 40 years of age, in cases of fall from standing height, it is more frequent in children and the elderly. A 64-year-old male patient, victim of a motorcycle accident, attended the Maxillofacial Surgery and Traumatology service at the Teresina Emergency Hospital. On clinical examination, he presented edema, lacerations and excoriations on the face on the right side, he presented a bone step on palpation of the mandible in the region of the right angle and symphysis. The image exam
\end{abstract}


showed, in the computed tomography, the presence of a discontinuity of the mandibular arch with multiple fracture lines, in the region of the right angle, right body and symphysis of the left mandible.Closing the diagnosis in Complex fracture of the mandible, thus forwarding the surgical intervention for reduction and fixation of the fracture. Four 2.0 system boards were used. Local infiltration with xylocaine with $2 \%$ vasoconstrictor was performed, incision for Risdon access (submandibular), fracture exposure, reanatomization of bone fragments and osteosynthesis with two 2.0 plates and 15 screws in the compression and tension area, the same was performed in the intraoral access in the symphysis region. Finally, suture by planes. The patient was discharged from the hospital within one day and continued with outpatient follow-up. Based on the case presented, in cases of mandible fractures, the extraoral risdon and intraoral access are effective andsafe.

Keywords: Mandibular fractures; Facial bones; Jaw fixation techniques.

\begin{abstract}
Resumen
La mandíbula es el único hueso móvil de la cara y tiene un papel en la masticación, la deglución, el habla y la estética facial. Sefractura con frecuencia, de origen variado, con una prevalencia de hasta los 40 años de edad, en los casos de caída desde la bipedestación, es más frecuente en niños y ancianos. Paciente masculino de 64 años, víctima de accidente de motocicleta, acudió al servicio de Cirugía y Traumatología Maxilofacial del Hospital de Urgencias Teresina. Al examen clínico presentaba edema, laceraciones y escoriaciones en la cara del lado derecho, presentaba un paso óseo a la palpación de la mandíbula en la región delángulo recto y sínfisis. El examen de imagen mostró, en la tomografía computarizada, la presencia de una discontinuidad del arco mandibular con múltiples líneas de fractura, en la región del ángulo recto, cuerpo derecho y sínfisis de la mandíbula izquierda. Cerrando el diagnóstico en Fractura compleja de mandíbula, adelantando así la intervención quirúrgica para la reducción y fijación de la fractura. Se utilizaron cuatro placas de sistema 2.0. Se realizó infiltración local con xilocaína con vasoconstrictor al 2\%, incisión para acceso de Risdon (submandibular), exposición de fractura, reanatomización de fragmentos óseos y osteosíntesis con dos placas 2.0 y 15 tornillos en el área de compresión y tensión, lo mismo se realizó en el acceso intraoral en la región de la sínfisis. Finalmente, sutura por planos. El paciente fue dado de alta del hospital en el plazo de un día y continuó con seguimiento ambulatorio. Con base en el caso presentado, en casos de fracturas de mandíbula, el acceso extraoraly el acceso intraoral son efectivos y seguros.
\end{abstract}

Palabras clave: Fracturas mandibulares; Huesos faciales; Técnicas de fijación mandibular.

\title{
1. Introdução
}

A mandíbula é um osso frequentemente fraturado em trauma maxilofacial, representando quase $15,5 \%$ a $59 \%$ de todas as fraturas faciais. A etiologia do trauma facial é heterogênea e o predomínio maior ou menor de um fator etiológico se relaciona com algumas características da população estudada (idade, sexo, classificação social, local, urbana e residencial). A literatura aponta que a idade do paciente traumatizado e a etiologia do trauma facial são fatores importantes na análise da casuística, incidindo, geralmente, entre jovens de até 40 anos e em decorrência de acidentes automobilísticos, seguido de traumas resultantes de práticas esportivas e agressões. Por outro lado, as fraturas relacionadas à queda da própria altura geralmente podem ser associadas a crianças e idosos, contudo, se mostraram menos frequentes, e a ocorrência pode ser justificada pela vigilância dos familiares nessa faixa etária. (Ahmedn et al, 2021).

Este componente é o único osso móvel da face e tem importante função na mastigação, deglutição, fonação e estética facial. Devido à sua topografia, anatomia e projeção anterior corporal é um osso muito exposto. Devido ao sítio anatômico composto, as abordagens cirúrgicas nesta área devem ter boa visualização e permitir que o cirurgião reduza precisamente a fratura e forneça fixação interna estável (Rodrigues et al, 2018).

Os sinais e sintomas mais associados às fraturas de mandíbula são limitação da abertura bucal, edema, assimetria facial, má oclusão, mobilidade atípica à manipulação, crepitação, parestesia e dor. A partir disso, compreende-se o manejo cirúrgico das fraturas consiste, basicamente, na redução e na fixação interna rígida dos cotos fraturados com auxílio de mini placas e parafusos. Esse tratamento é largamente indicado em casos de fraturas complexas e desfavoráveis da mandíbula. A fixação desses cotos busca reduzir a mobilidade da fratura, impedindo a formação de tecido de interposição entre os ossos que dificulte sua cicatrização, quadro denominado pseudoartrose (Rodrigues et al, 2018).

Com isso, o objetivo deste trabalho consiste em descrever, por meio de um relato de caso, uma fratura de mandíbula em região de ângulo direito e parassínfise, causada por acidente motociclístico tratada pelo serviço de Cirurgia e Traumatologia 
Bucomaxilofacial do Hospital de Urgência de Teresina (HUT).

\section{Metodologia}

Desenvolveu- se este estudo de caso, a partir de um processo de avaliação de exames clínicos, anamnese, exame físico e exame de imagem (Tomografia Computadorizada). Associado a este levantamento das informações do caso, foi realizada uma busca literária nos bancos de dados como Pubmed, Scielo e CAPES, realizando uma análise dos artigos que estavam vinculados à temática do estudo e de acordo com as palavras-chave utilizados no trabalho. $\mathrm{O}$ trabalho preserva a identidade do paciente $\mathrm{e}$ foi assinado O Termo de Consentimento Livre e Esclarecido - TCLE.

\section{Relato de Caso}

Paciente do sexo masculino, 64 anos de idade, vítima de acidente automobilístico, compareceu ao serviço de emergência de Cirurgia e Traumatologia Bucomaxilofacial do Hospital de Urgência de Teresina - HUT, apresentando trauma em face, foi solicitado parecer clínico das equipes da neurocirurgia e Cirurgia e Traumatologia Bucomaxilofacial, para avaliação secundária do paciente.

A equipe de Cirurgia e Traumatologia Bucomaxilofacial no momento do atendimento constatou que apresentava edema, lacerações e escoriações na face no lado direito, estava consciente, orientado, eupneico em ar ambiente, apresentou degrau ósseo à palpação de mandíbula em região de ângulo direito e sínfise. O paciente não tinha os elementos dentários 11, 12,13, 21, 22 ou desdentado parcial, sendo possível visualizar desnivelamento oclusal e mobilidade sem exposição óssea. Não se encontraram alterações em terço médio e superior de face.

Para fechar o diagnóstico e melhor visualizar a fratura, foram solicitados exames de imagem. Constatou-se, na tomografia computadorizada de face, a presença de uma imagem sugestiva de descontinuidade do arco mandibular com múltiplos traços de fratura, em região de ângulo direito, corpo direito e sínfise da mandíbula esquerda (Figura 1). Para regressão do quadro de edema foi administrada Dexametasona de $4 \mathrm{mg}$ de $8 / 8$ horas, redução de foco infeccioso administrando- se Cefazolina $1 \mathrm{~g}$ EV de $8 / 8$ horas e Tramadol 50mg EV para controle de analgesia.

Após alta clínica por parte das outras especialidades, a equipe da Bucomaxilo planejou intervenção cirúrgica. Para tal, foram solicitados exames pré-operatórios (hemograma, tempo de tromboplastina parcial, tempo de tromboplastina parcial ativado, ureia, creatinina, glicemia em jejum), sendo, então, o tratamento cirúrgico indicado com planejamento para redução e fixação da fratura com miniplacas e parafusos, sob anestesia geral e intubação nasotraqueal.

Foi optado pela realização de dois tipos de acessos: acesso submandibular e acesso intra-oral em fundo de vestíbulo maxilar, utilizando quatro placas do sistema 2.0 através do acesso submandibular. Primeiro, realizou-se a infiltração local com xilocaína com vasoconstritor $2 \%$, incisão com bisturi elétrico para o acesso de Risdon (submandibular), exposição da fratura (Figuras 2,3), reanatomização dos fragmentos ósseos, após isso, fora feito a realização do acesso em fundo de sulco para exposição das fraturas osseas em região parassinfisaria. Logos após, estabilizou-se os fragmentos ósseos, analisou a questão da oclusão do paciente e se realizou a fixação com placas e parafusos do sistema 2.0mm (Figuras 4, 5).

Procedeu-se com lavagem copiosa com soro fisiológico $0,9 \%$ e fechamento dos acessos cirúrgicos, o extraoral por planos com monocryl 4-0 e sutura em pele com fio nylon 5-0, já para o acesso intraoral foi apenas utilizado vicryl 4-0.

O paciente teve alta um dia após procedimento cirúrgico com retorno ao ambulatório Bucomaxilofacial para acompanhamento, com prescrição domiciliar de Cefazolina 500mg tomar um comprimido de $8 \mathrm{~h} / 8 \mathrm{~h}$ horas por sete dias,

Diclofenaco 50mg tomar um comprimido de 6h/6h durante 3 dias, Dipirona Sódica 500mg tomar um comprimido de $6 \mathrm{~h} / 6 \mathrm{~h}$ horas dia por três dias, bochecho com Clorexidina $0,12 \% 10 \mathrm{ml} 8 \mathrm{~h} / 8 \mathrm{~h}$ por sete dias. Em radiografia pós-operatória observou-se satisfatória redução da fratura (Figura 6). O pós-operatório evoluiu com ótima cicatrização, sem edema, 
Research, Society and Development, v. 10, n. 17, e172101721697, 2021

(CC BY 4.0) | ISSN 2525-3409 | DOI: http://dx.doi.org/10.33448/rsd-v10i17.21697

restabelecendo as funções de fonação, mastigação e boa abertura bucal.

Figura 1. Tomografia computadorizada pré-operatório.

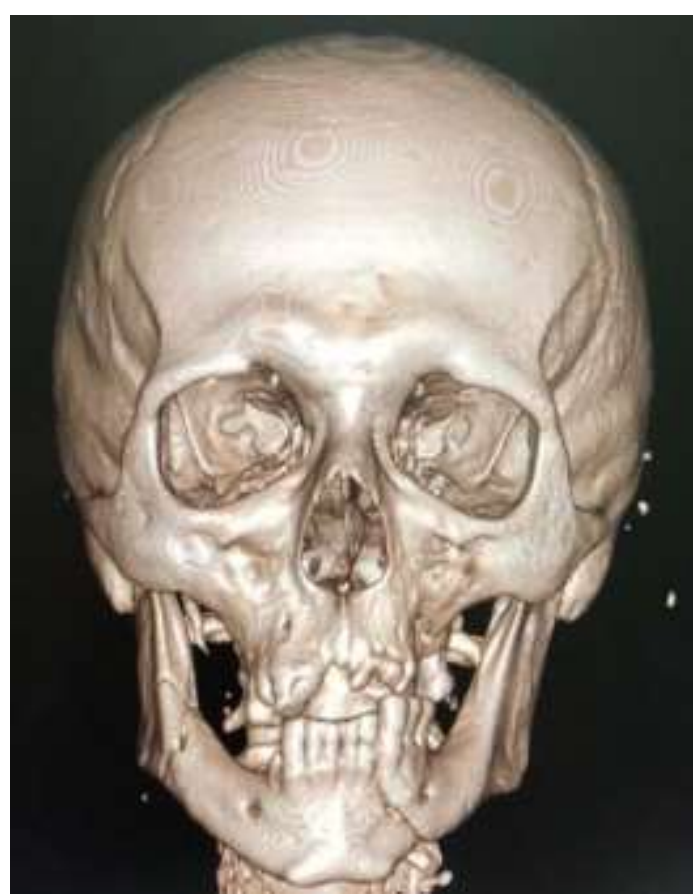

Fonte: Autores.

Figura 2. Tomografia computadorizada pré-operatório.

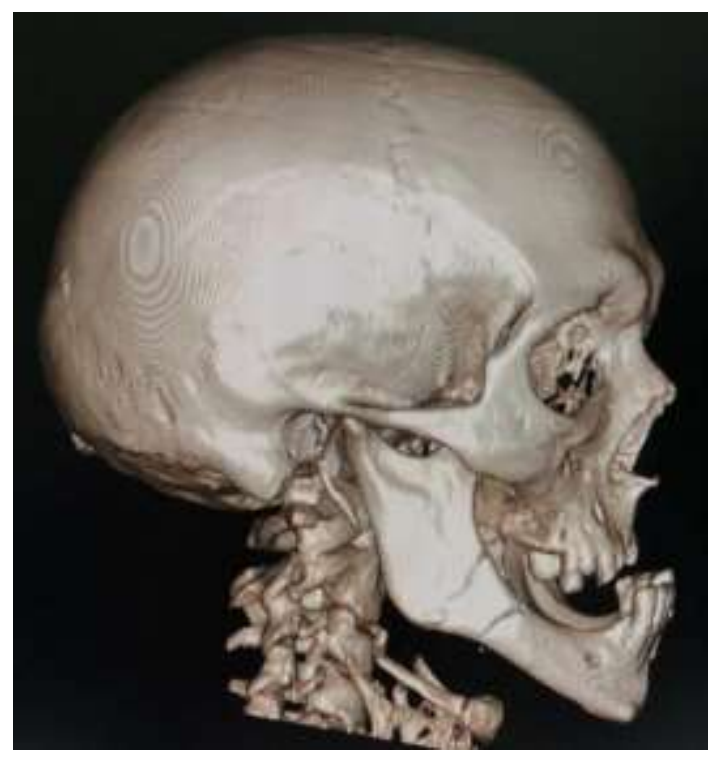

Fonte: Autores. 
Research, Society and Development, v. 10, n. 17, e172101721697, 2021

(CC BY 4.0) | ISSN 2525-3409 | DOI: http://dx.doi.org/10.33448/rsd-v10i17.21697

Figura 3. Abordagem cirúrgica à região submandibular direita.

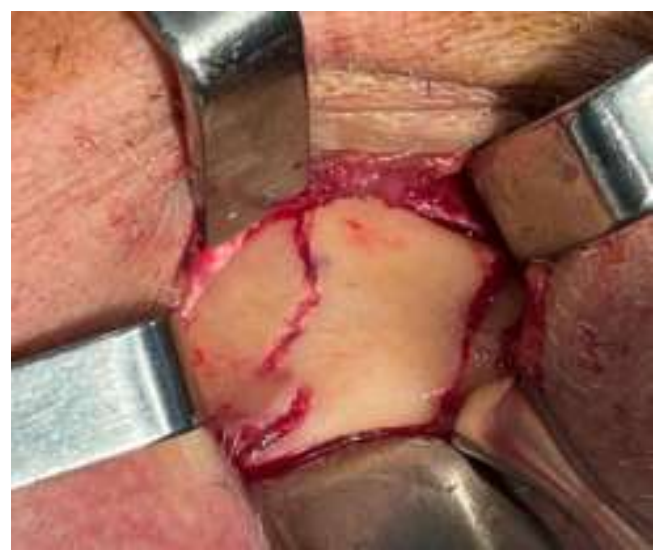

Fonte: Autores.

Figura 4. Abordagem cirúrgica na região de parassínfise esquerda.

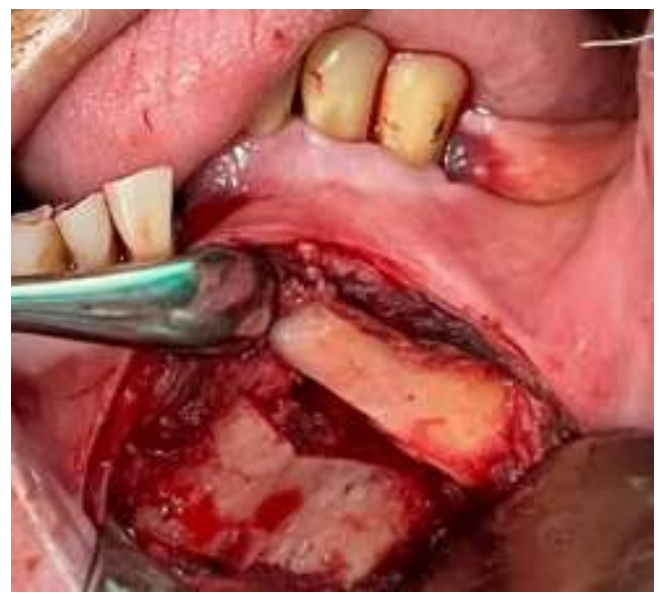

Fonte: Autores.

Figura 5. Osteossíntese com placas e parafusos do sistema 2.0 em ângulo de mandíbula direita.

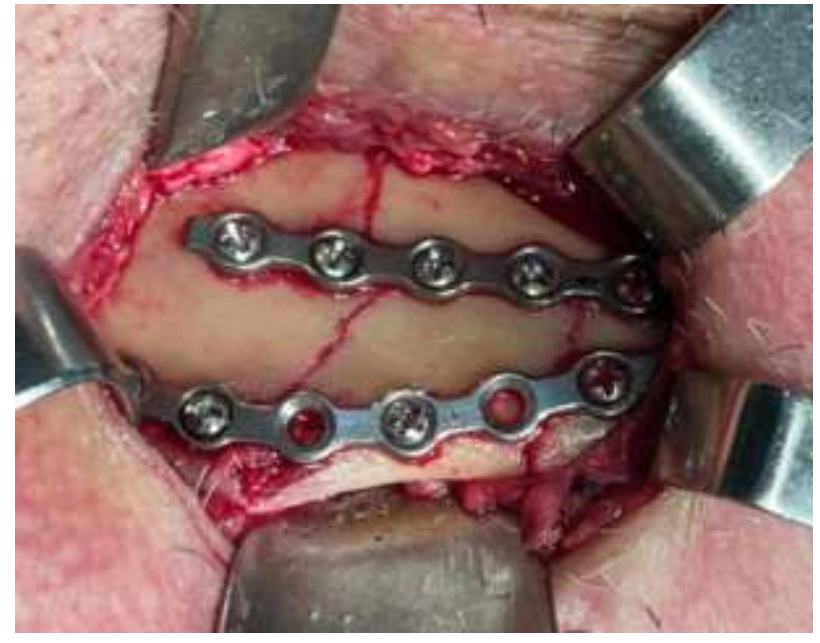

Fonte: Autores. 
Research, Society and Development, v. 10, n. 17, e172101721697, 2021

(CC BY 4.0) | ISSN 2525-3409 | DOI: http://dx.doi.org/10.33448/rsd-v10i17.21697

Figura 6. Osteossíntese com placas e parafusos do sistema 2.0 em sínfise mandibular esquerda.

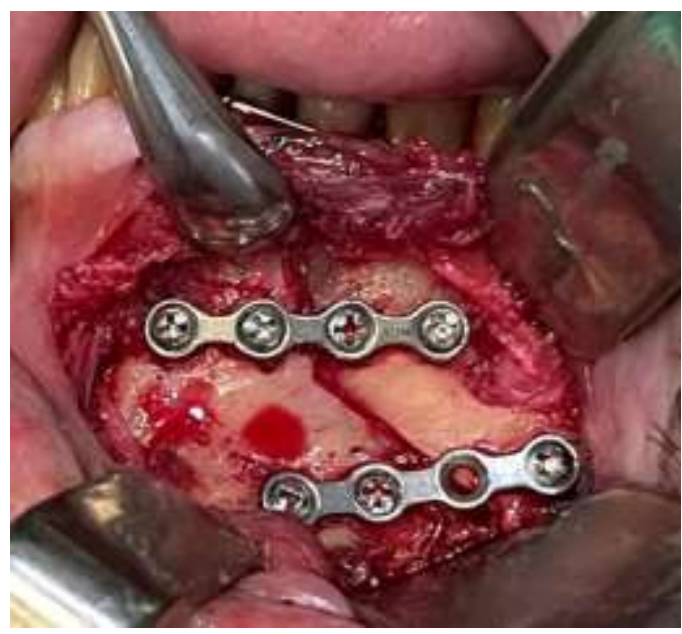

Fonte: Autores.

Figura 7. Tomografia computadorizada do pós-operatório imediato.

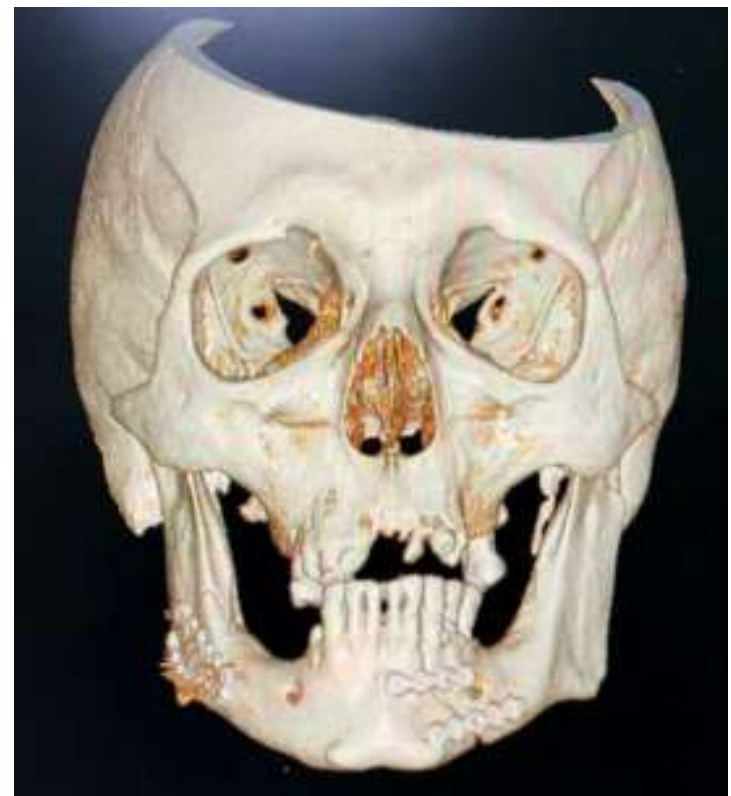

Fonte: Autores. 
Figura 8. Tomografia computadorizada do pós-operatório imediato.

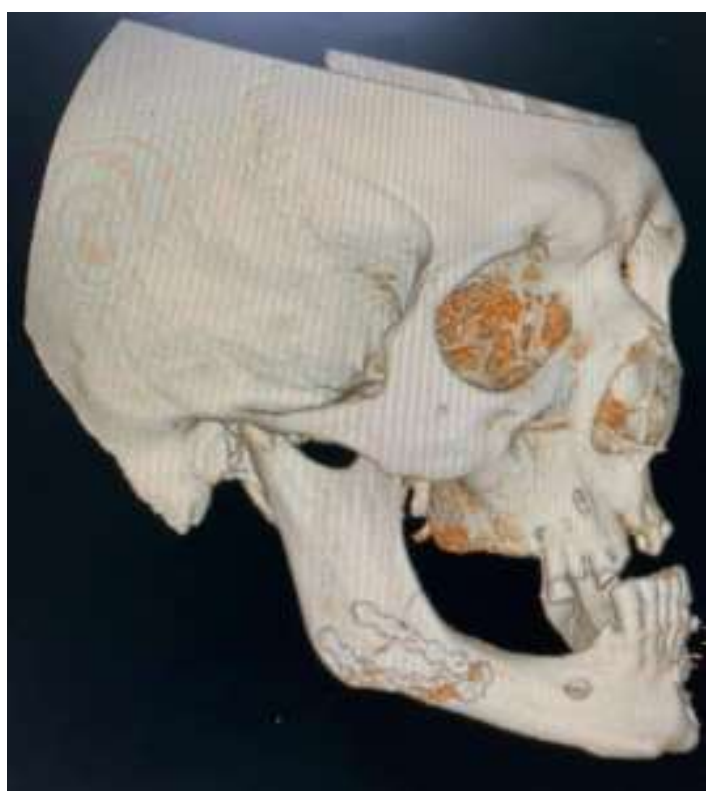

Fonte: Autores.

\section{Resultados e Discussão}

As fraturas de mandíbula são habituais entre cirurgiões bucomaixlofaciais e tendem a se estender em áreas adversas deste componente ósseo. Elas podem se estender por regiões (ramo, ângulo, corpo, parassínfise e sínfise), envolvendo regiões e estruturas vizinhas não usuais. Quando se trata de fraturas múltiplas, a classificação se trata em um tipo especial, necessitando, assim, um planejamento, até mesmo em ambientes hospitalares de urgência, por conta do nível de complexidade.A força necessária para gerar esse tipo de fratura, parece ser resultante da aplicação de força em um determinado tempo em local único, gerando energia necessária para provocar a alteração da arquitetura óssea mandibular. Impactos com alta energia levam à concentração de energia na mandíbula, causando fraturas múltiplas. No caso relatado, o paciente foi vítima de acidente motociclistico, apresentando fraturas múltiplas na região de ângulo e parasínfise esquerda. (Rodrigues et al, 2018).

Para a maioria dos cirurgiões a mandíbula consiste no alicerce para o restabelecimento da oclusão em primeiro lugar, pois a mandíbula é o osso mais resistente da face e pode ser anatomicamente reduzida mais facilmente que a maxila daí a preferência pela sequência "de baixo para cima e de dentro para fora". Com a mandíbula reconstruída, irá restabelecer a largura, projeção e altura facial posterior da porção inferior da face.Com relação ao tratamento das fraturas mandibulares o acesso ideal deve permitir a redução adequada, a fixação com placas e parafusos e evitar morbidade, principalmente relativa ao nervo facial e seus ramos , no entanto, tem o inconveniente de propiciar a cicatriz externa fato que não ficou evidente no caso apresentado, demonstrando bom aspecto da cicatrização e ausência de paralisia do nervo facial (Mendonça et al, 2015).

$\mathrm{O}$ acesso submandibular (ou de Risdon), está indicado para as fraturas de corpo mandibular e ângulo viabilizando a redução anatômica; além disto, apresenta baixo índice de contaminação, por não haver, a rigor, comunicação com o meio bucal e o amplo acesso que favorece a adaptação dos diversos tipos de fixação rígida, dentre outros. Associado a ele, foi realizado o acesso intraoral em fundo de sulco para exposição da fratura parassinfisaria, atendendo a necessidade cirurgia diante de uma cirurgia complexa com fraturas múltiplas. Além da excelência quanto a técnica cirúrgica, é importante salientar quanto as estruturas nobres que circundam as regiões trabalhadas, visto isso, o conhecimento e capacitação quanto a anatomia é crucial (Rodrigues et al, 2017).

O objetivo final e os critérios para o procedimento cirúrgico são promover uma boa adaptação e estabilidade dos óssea, 
portanto, a escolha da técnica é fundamental, sendo o acesso submandibular e a osteossíntese com fixação interna rígida, são ótimas opções, minimizando as chances de deslocamento da fratura de mandíbula em região de ângulo e sínfise.

\section{Considerações Finais}

A partir do caso clínico apresentado, em casos de fraturas complexas de mandíbula o acesso extra oral de ridson se mostra eficaz e seguro no tratamento destas fraturas em região de ângulo, com objetivo de restabelecer as funções mastigatórias. Não houve lesão do ramo marginal do nervo facial bem como também não houve lesão do nervo alveolar. Cicatrização tecidualboa dentro da idade e satisfação estética e funcional do paciente.

\section{Referências}

Bartoli, M. M., Eidt, J. M. S., Engelmann, J. L., Rocha, F. D., \& Conto, F. (2014) Trauma maxilofacial: avaliação de 1385 casos de fraturas deface na cidade de Passos Fundo - RS. Rev. Cir. Traumatol. Buco-Maxilo-Fac. 14(2):87-94.

Cavalcante JR., Guimarães, K. B., Vasconcelos, B. C. E. \& Vasconcellos, R. J. H. (2009). Estudo epidemiológico dos pacientes atendidos com trauma de face no hospital Antônio Targino-Campinas Grande/Paraíba. Brazillian Journal of Otorhinolaryngology. 75(5), 628-33.

Custódio, A. L. N., Menezes Jr, D. C., Cavalcanti, F. M. N., Serpa, M. R., Cosso, M. G. \& Faria, J. M. P. (2007). Consideraçõessobre o tratamento de fratura mandibular após remoção de terceiro molar. Arq Bras Odontol. 3(2),106-13.

Chaly a, P. L., Mabula, J. B., Ngayomela, I. H., Kanumba, E. S., Chandika, A. B., Giiti, G., \& Balamuka, D. (2010). Motorcycle injuries as an emerging public health problem in Mwanza City, Tanzania: A call for urgent int ervention. Tanzania Journal of Health Research , 12 (4), 214 - 221.

Fritz M. A. K. P. J. (2002). Sequencing And Organization Of The Repair Of Panfacial Fractures. Oper Tec Otolaryngol Head Neck Surg. ;13(4):261-4.

Gaetti Jardim, E. C., Faverani, L. P., Ramalho-Ferreira, G., Pereira, C. C. S., Gealh, W. C., \& Shinohara, E. H. (2011). Acessos Cirúrgicos A Articulação Temporomandibular: Revisão De Literatura. Revista Sbccp; 40(1): 46-52.

Giacomin M., Conto F. D., Siqueira S. P., Signori P. H. , Eidt J. M. S., \& Sawazaki R. (2017). Trauma facial em idosos: umaanálise retrospectiva de 10 anos. Rev. bras. geriatr. gerontol. 20(5), $618-623$.

Mendonça J. C. G., Jardim, E. C. G., Manrique, G. R., Lopes, H. B., \& Freitas, G. P. (2012). Tratamento De Fratura Complexa De Corpo MandibularPor Meio De Acesso Submandibular. Revista Sbccp;41(1):33-5

Mendonça, J. C. C., et al. (2015). Fernando Ribeiro Teixeira. Access For Extraoral Angle Fracture Osteosynthesis Mandibular. ArchHealth Invest 4(6): 9-14

Mendonça, J. C. G., Gaetti - Jardim, E. C., Manrique, G. R., Lopes, H. B., \& Freitas, G. P. (2013). Acesso Cirúrgico Para Tratamento De Fraturas Mandibulares: Revisão De Literatura. Arch Health Invest;2(2):19-23.

Minari , I. S., Figueiredo, C. M. B. F., de O liveira, J. C. S., Brandini, D. A., \& Bassi, A. P. F. (2020). Incidência de múltiplas fraturas faciais: estudo retrospectivo de 20 anos. Research, Society and Development, 9(8), e327985347 - e327985347.

Patrocínio L. G., Patrocínio J. A., Borba B. H. C.,Bonatti B. S., Pinto L. F., Vieira J. V., \& Costa J. M. C. (2005). Fratura De Mandíbula: Análise De 293 Pacientes Tratados No Hospital De Clínicas Da Universidade Federal De Uberlândia. Rev Bras Otorrinolaringol;71 (5); $560-65$.

Pereira, A. S., Shitsuka, D. M., Parreira, F. J., \& Shitsuka, R. (2018). Metodologia em pesquisa científica. UFSM.

Rodrigues, R. D., et al. (2017). Surgical Management Of Mandibular Fracture: Case Report. Rfo Upf, 23(3), 343-347.

Rodrigues, C. M. C., et al. (2018). Surgical Approach Of Complex Facial Fractures: The Importance Of Sequential Planning. RelatosCasos Cir.;4(4):E1993

Shaheen A .(2021). The Retromandibular Transparotid Approach Vs. Retromandibular Retroparotid Approach For The Mandibular Condyle: Our Clinical Experience Braz. J. Oral Sci; 20: E211443.

Silva, L. F., Borbosa, C. H. D., Mesquita, L. V., Barbalho, J. C. M., Carvalho, A. C. G. S., \&Mello, M. J. R. (2014) .Epidemiologia Dos Traumatismos De Face Em Pacientes Jovens No Estado Do Ceará. Rev. Cir. Traumatol. Buco-Maxilo-Fac. 14(3):79-84.

Silva, J., \& Caúas, M. C. (2004). Fratura De Mandíbula Decorrente De Acidente Automobilístico: Relato De Caso. Odontologia. Clín. Científ. Recife; 3(3):199 - 208.

Tullio A, \& Sesenna E. (2000). Role Of Surgical Reduction Of Condylar Fractures In The Management Of Panfacial Fractures. BrJ Oral Maxillofac Surg;38 (5):472-6.

Wulkan, M., Parreira Jr, J. G., \& Botter, D. A. (2005). Epidemiologia do trauma facial. Revista da associação médicabrasileira, 51 (5), 290 - 295. 